\title{
The First Global Physical Activity and Sedentary Behavior Guidelines for People Living With Disability
}

\author{
Catherine Carty, Hidde P. van der Ploeg, Stuart J.H. Biddle, Fiona Bull, Juana Willumsen, Lindsay Lee, \\ Kaloyan Kamenov, and Karen Milton
}

\begin{abstract}
Background: The World Health Organization has released the first global public health guidelines on physical activity and sedentary behavior for people living with disability. This paper presents the guidelines, related processes, and evidence, and elaborates upon how the guidelines can support inclusive policy, practice, and research. Methods: Methods were consistent with the World Health Organization protocols for developing guidelines. Systematic reviews of the evidence on physical activity for health for people living with disability were appraised, along with a consideration of the evidence used to inform the general 2020 World Health Organization guidelines. Results: Evidence supported the development of recommendations for people living with disability, stressing that there are no major risks to engaging in physical activity appropriate to an individual's current activity level, health status, and physical function, and that the health benefits accrued generally outweigh the risks. They also emphasize the benefits of limiting sedentary behavior. Conclusions: The guidelines mark a positive step forward for disability inclusion, but considerable effort is needed to advance the agenda. This paper highlights key considerations for the implementation of the new recommendations for people living with disability, in line with the human rights agenda underpinning the Global Action Plan on Physical Activity 2018-2030 and allied policies.
\end{abstract}

Keywords: recommendations, public health, mainstream inclusion, sustainable development, human rights

Disability "is part of the human condition" that most people will experience to varying degrees in their lifetime, ${ }^{1(\mathrm{p} 3)}$ and it impacts opportunities to engage in physical activity. Disability can be understood as an interaction between personal, biological, societal, and environmental factors that can prevent "full and effective participation in society on an equal basis with others." $\left.{ }^{4} 4\right)_{2-4}$ Disability can be represented on a continuum, relevant to all, underlining the need for societies to mainstream disability in all sectors. ${ }^{1,5}$ Disability is a global public health and human rights issue, with 1.5 billion people currently living with disability worldwide. ${ }^{1,6}$ People with disability face barriers in accessing health services and experience stigmatization, discrimination, and rights violations, ${ }^{4}$ leading to social, economic, and health marginalization. ${ }^{7}$ Globally, over the last 30 years, the total burden of disability, driven in large part by the inclusion of conditions associated with noncommunicable disease, increased by $52 \% .{ }^{8}$ This can be reversed, and health gains made, by attending to and resourcing the drivers of health, ${ }^{8}$ including physical activity.

Carty is with the UNESCO Chair, "Transforming the Lives of People with Disabilities, their Families and Communities, Through Physical Education, Sport, Recreation and Fitness," Munster Technological University, Tralee, Ireland. Van der Ploeg is with the Department of Public and Occupational Health, Amsterdam Public Health Research Institute, Amsterdam UMC, Vrije Universiteit Amsterdam, Amsterdam, The Netherlands; and the Sydney School of Public Health, The University of Sydney, Sydney, NSW, Australia. Biddle is with the Centre for Health Research, University of Southern Queensland, Springfield, Australia. Bull and Willumsen are with the Physical Activity Unit, Department of Health Promotion, World Health Organization, Geneva, Switzerland. Lee and Kamenov are with the Sensory Functions, Disability and Rehabilitation Unit, Department of Noncommunicable Diseases, World Health Organization, Geneva, Switzerland. Milton is with the Norwich Medical School, Faculty of Medicine and Health Sciences, University of East Anglia, Norwich, United Kingdom. Carty (catherine.carty@ittralee.ie) is corresponding author.
Disability negatively impacts opportunities to gain health benefits, mitigate health risks, and improve health outcomes through physical activity and limiting sedentary behavior. People living with disability are at least twice as likely to be physically inactive as those without disability, ${ }^{9}$ increasing the risk of noncommunicable diseases and comorbidities, while also being potentially detrimental for mental health and social well-being. 1,9,10 Lower participation reflects additional barriers faced by people with disability, including physical, personal, social, and environmental barriers. ${ }^{11,12}$ Creating opportunities for inclusion in physical activity for people living with disability can help eliminate such barriers by changing perceptions, emphasizing strengths and abilities, promoting personal resilience, and having an onward impact on inclusion in society. ${ }^{7}$

In 2015, the United Nations launched "Transforming Our World: The 2030 Agenda for Sustainable Development."13 Its vision of "no-one left behind," starting with the most vulnerable, put a focus on disability inclusion, which has subsequently been embraced in key policy developments in physical activity and sport. ${ }^{12,14,15}$ Disability has been recognized by the World Health Organization (WHO) as a development priority. ${ }^{12}$ The publication of the first WHO guidelines on physical activity and sedentary behavior for people living with disability ${ }^{16}$ reflects the WHO's commitment to inclusive actions, aligned with the 2030 Agenda and expressed in the Global Action Plan on Physical Activity 2018-2030. ${ }^{12}$ Incorporating equity and human rights is integral to the WHO guideline development process. ${ }^{17}$ The emergence of these public health recommendations for people living with disability facilitates the development and implementation of related policies, research, and practices that can reduce discrimination and create opportunities for inclusive physical activity participation and better health outcomes among this population.

The aims of this paper are to (1) summarize the process and evidence that informed the first global guidelines on physical 
activity and sedentary behavior for people living with disability, (2) present the guidelines, (3) discuss how these guidelines can support policy implementation in physical activity and sedentary behavior in the context of human rights and disability inclusion, and (4) present 10 areas for advancing inclusive practice in physical activity and sedentary behavior for people living with disability.

\section{Methods}

In 2019, the WHO commenced work to update the 2010 Global Recommendations on Physical Activity for Health. ${ }^{18}$ A Guideline Development Group (GDG) was established, consisting of 27 experts from relevant scientific disciplines, including the disability area, as well as practitioners and decision makers in the field, representing all regions. The WHO steering group was composed of staff from different areas of the organization and included a disability expert, who also lives with disability. Public consultation on the draft guidelines was conducted. This was widely circulated to organizations and individual researchers in the field of disability and physical activity. The WHO guidelines were developed in accordance with the WHO Handbook for Guideline Development, ${ }^{17}$ and details of the methodology can be found elsewhere. ${ }^{16,19}$

The scientific report of the Physical Activity Guidelines Advisory Committee ${ }^{20}$ provides a summary of evidence on physical activity and sedentary behavior for health outcomes from 2008 to 2016, including disability. For the purposes of developing the new WHO guidelines, this review was updated through a search for systematic reviews and pooled analyses of cohort studies published from 2017 up to September 2019.

Evidence on the association between physical activity and health for 8 specific conditions that can lead to disability in children, adolescents, and adults were included: multiple sclerosis, spinal cord injury, intellectual disability, Parkinson's disease, stroke, schizophrenia, major clinical depression, and attention deficit hyperactivity disorder. Four health outcomes were considered across the 8 health conditions, namely, comorbidities, physical function, cognitive function, and quality of life, and are summarized in Table 1. The Grading of Recommendations Assessment, Development and Evaluation method was used to rate the certainty of the evidence for each outcome. ${ }^{21}$

Given the dearth of research assessing the associations between physical activity and the critical health outcomes of cardiovascular disease mortality, incident hypertension, incident site-specific cancers, and incident type 2 diabetes, the GDG assessed the scientific evidence used to inform the development of recommendations for age-specific population groups in adults and older adults. Similarly, the scientific evidence collated for the development of recommendations for children and adolescents was also reviewed. The GDG considered the applicability of the broader evidence to children and adults living with disability and the appropriateness of extrapolation to support the development of recommendations. Full details of the critical and important outcomes that were assessed in relation to the age-specific population recommendations are documented in detail elsewhere. ${ }^{16}$

Where extrapolation from the general age-group guidelines was considered appropriate, the evidence rating was downgraded due to indirectness. In addition to the evidence, the following were also considered when developing the recommendations for consideration by the WHO: the benefits and harms, values and preferences of those affected by the guidelines, the resource implications of the recommendations, the impact on health equity, and the acceptability and feasibility of the recommendations.

\section{Results}

From the evidence considered by the PAGAC and 187 new reviews identified, 101 reviews met the inclusion criteria to inform the development of specific recommendations for consideration by the WHO. A key reason for exclusion was that many studies involving people living with disability centered on fitness and functional outcomes as part of clinical care and rehabilitation; these studies were not deemed relevant, given the "public health" focus of the guidelines. The volume of evidence varied by condition, from the highest number of reviews for multiple sclerosis $(n=28)$, followed by Parkinson's $(n=24)$, history of stroke $(n=15)$, spinal cord injury $(n=13)$, intellectual disability $(n=7)$, Schizophrenia $(n=6)$, major clinical depression $(n=5)$, and attention deficit hyperactivity disorder $(n=3)$. The included reviews covered a broad range of study designs, including randomized controlled trials, nonrandomized controlled studies, before and after studies, case controls, case reports/case studies, and observational studies.

The certainty of the evidence across conditions ranged from low to high. There were no studies found on physical activity and comorbidity in people living with multiple sclerosis and insufficient evidence to inform a judgment on the association with comorbidity and quality of life in people with intellectual disability. A summary of the evidence available for each specific health condition and outcome is provided in Table 2 .

When considering the critical and important health outcomes assessed in the development of the general age-specific recommendations, this included assessing whether there was any evidence or reasons that the findings would not apply to people living with disability. The GDG concluded, based on expert opinion, that the associations between physical activity and sedentary behavior on selected key outcomes could be expected to result in the same health benefits for people living with disability as the general

Table 1 Critical Outcomes Considered by Health Condition and Number of Reviews

\begin{tabular}{lccc}
\hline Health condition & Comorbidity & Physical function & Quality of life \\
\hline Multiple sclerosis & 0 & 15 & 12 \\
Spinal cord injury & 3 & 8 & 2 \\
Intellectual disability & 1 & 5 & 1 \\
Parkinson's disease & & 22 & 14 \\
Stroke & & & 1 \\
Schizophrenia & & 2 \\
Major clinical depression & & 5 \\
Attention deficit hyperactivity disorder & & 3 \\
\hline
\end{tabular}


Table 2 Summary of the Evidence on Health Outcomes for Each Condition Group

\begin{tabular}{|c|c|}
\hline $\begin{array}{l}\text { Condition and health } \\
\text { outcome }\end{array}$ & Description of certainty of evidence by disability and health outcome \\
\hline \multicolumn{2}{|l|}{ Multiple sclerosis } \\
\hline Comorbidity & Not assignable \\
\hline Physical function & $\begin{array}{l}\text { High-certainty evidence shows aerobic and muscle-strengthening activities that improve physical function; functional } \\
\text { mobility; walking speed and endurance; and cardiorespiratory fitness, strength, and balance }\end{array}$ \\
\hline Quality of life & $\begin{array}{l}\text { Low-certainty evidence of improved quality of life, including symptoms of fatigue and depressive symptoms among } \\
\text { adults }\end{array}$ \\
\hline Cognition & Moderate-certainty evidence of a beneficial effect on cognition \\
\hline \multicolumn{2}{|l|}{ Spinal cord injury } \\
\hline Comorbidity & Low-certainty evidence that physical activity reduces shoulder pain and improves vascular function in paralyzed limbs \\
\hline Physical function & Moderate-certainty evidence shows improved walking function, muscular strength, and upper-extremity function \\
\hline Quality of life & Low-certainty evidence that physical activity enhances health-related quality of life \\
\hline \multicolumn{2}{|l|}{ Intellectual disability } \\
\hline Comorbidity & Not assignable \\
\hline Physical function & Low-certainty evidence of improved physical function in children and adults \\
\hline Quality of life & Not assignable \\
\hline \multicolumn{2}{|l|}{ Parkinson's disease } \\
\hline Physical function & High-certainty evidence of improvement in walking, balance, strength, and disease-specific motor scores \\
\hline Cognition & $\begin{array}{l}\text { Moderate-certainty evidence indicates that moderate to vigorous physical activity can have beneficial effects on } \\
\text { cognition }\end{array}$ \\
\hline \multicolumn{2}{|l|}{ Stroke } \\
\hline Physical function & $\begin{array}{l}\text { Moderate-certainty evidence for improved gait speed and ability, walking speed, distance and endurance, cardiore- } \\
\text { spiratory fitness, upper-limb function, sensory motor function of the lower limb, balance, mobility, and activities of daily } \\
\text { living }\end{array}$ \\
\hline Cognition & Moderate-certainty evidence of beneficial effects on cognition \\
\hline \multicolumn{2}{|l|}{ Schizophrenia } \\
\hline Quality of life & Moderate-certainty evidence indicates improved quality of life \\
\hline Cognition & $\begin{array}{l}\text { High-certainty evidence that moderate to vigorous physical activity can have beneficial effects on cognition, working } \\
\text { memory, social cognition, and attention/vigilance }\end{array}$ \\
\hline \multicolumn{2}{|l|}{ Major clinical depression } \\
\hline Quality of life & Moderate-certainty evidence that physical activity improves quality of life in adults \\
\hline \multicolumn{2}{|l|}{$\begin{array}{l}\text { Attention deficit } \\
\text { hyperactivity disorder }\end{array}$} \\
\hline Cognition & $\begin{array}{l}\text { Moderate-certainty evidence that moderate to vigorous physical activity can have beneficial effects on cognition, } \\
\text { including attention, executive function, and social disorders }\end{array}$ \\
\hline
\end{tabular}

population. For youth, this included favorable outcomes on cardiorespiratory and muscular fitness, cardiometabolic health, bone health, cognitive outcomes, mental health, and adiposity; for adults, this included reduction in all-cause mortality, cardiovascular mortality, incident hypertension, incident site-specific cancers, incident type 2 diabetes, improved mental health, cognitive health and sleep, and possible improvements in adiposity; and for older adults, this included the additional benefits of prevention of falls and falls-related injuries and favorable outcomes on bone health and functional ability. As a result, the physical activity recommendations drafted for children and adolescents and for adults were adopted and applied to people living with disability.

The recommendations were also deemed applicable to people living with disability broadly, including those living with disability arising from health conditions that were not considered in this review. Chronic conditions are addressed separately in both the guidelines and associated publications. ${ }^{16,22}$ In many instances, the impairment rather than the health condition per se can impact the exercise response. People living with Parkinson's or a history of stroke (evidence for which was reviewed) can experience difficulties with mobility, motor control, tremors, and paralysis in limbs, which could also be experienced by people living with muscular dystrophy or cerebral palsy. In the case of other disability areas, for example, visual or hearing impairments, while specific evidence was not reviewed, there is no physiological reason to assume different health outcomes from physical activity participation or limiting sedentary behavior. However, due to the indirectness of the evidence, the certainty of the evidence was downgraded to reflect the extrapolation. The GDG noted specifically that resultant recommendations were applicable for those living with disability "where possible and as able," to recognize and acknowledge the heterogeneity of this population. Consistent with the recommendations developed for other population groups, the GDG concluded that some physical activity is better than none, and that people living with disability should start with small amounts of physical activity and gradually increase the frequency, intensity, and duration over time where able.

Due to the lack of evidence on sedentary behavior and health outcomes among people living with disability, the evidence from the 
general population was considered. The GDG concluded that there was no reason to believe that the health outcomes of limiting sedentary behavior would be any different in people living with disability. It was therefore agreed, based on expert opinion, that the evidence on sedentary behavior and countering high levels of sedentary behavior through undertaking lighter and more moderate to vigorous physical activity from general populations could be extrapolated to people living with disability. The certainty of the evidence was downgraded due to indirectness. Replacing sedentary behavior with light-intensity physical activity is especially important for people who are the least active and people with mobility impairments who spend much or all day sitting or lying down and for whom moderateto vigorous-intensity physical activity may present a challenge.

The guidelines stress that there are no major risks to people living with disability engaging in physical activity when it is appropriate to an individual's current activity level, health status, and physical function and that the health benefits accrued generally outweigh the risks. Importantly, all physical activity counts, including light-intensity physical activity. The new WHO guidelines for children and adolescents with disability and adults with disability are shown in Boxes 1 and 2, respectively.

\section{Discussion}

The publication of the WHO guidelines on physical activity and sedentary behavior for people living with disability bridges a gap between policy aspirations and practical implementation. They affirm the associated health benefits, quantify the related volume and type of activity necessary to achieve the benefits, and provide good practice suggestions. This set of recommendations gives clarity to those designing and delivering public health physical activity interventions and advocacy messages on the why, what, and how people with disability should engage in physical activity and limit sedentary behavior.

The emergence of these guidelines marks a purposeful and positive step toward including people living with disability in mainstream physical activity initiatives, thereby advancing their human rights and mainstreaming disability inclusion. Human rights approaches underpin the Global Action Plan on Physical Activity 2018-2030, the Kazan Action Plan and the United Nations Action Plan on Sport for Development and Peace. ${ }^{12,14,15}$ All 3 action plans acknowledge the inequity in access to physical activity for people living with disability. These complementary instruments, together with their follow-up implementation initiatives, have stimulated input from many stakeholders, including academia, designed to eliminate barriers and universalize access to physical activity. ${ }^{15}$ The new guidelines will support advancing an inclusive agenda and the increasing acceptance that inclusion not only benefits people living with disability, but also their families, communities, and all members of society.

The evidence-based guidelines bring clarity to physical activity and sedentary behavior for people living with disability. The capacity to combine the evidence base for those living with disability with evidence extrapolated from the general population emphasizes the homogeneity in the physiological response to physical activity and sedentary behavior, regardless of impairment. Where condition-specific evidence was not reviewed (eg, autism, spina bifida), many functions affected by these conditions were addressed in the evidence, enabling broader consideration of the evidence. As previously mentioned, in the case of other disability areas (eg, visual or hearing impairments), where there was no physiological reason to assume different health outcomes, the opportunity to accrue health outcomes associated with physical activity and limiting sedentary behavior relates more to factors beyond impairment or health conditions alone.

While the evidence base for the general age-specific populations was also applied to those with impairments in order to increase the total volume of evidence considered, this does not mitigate the need to increase the volume of disability-specific research in the future. Physical activity outcomes and side effects may vary across different impairment groups in ways that have not yet been studied. New research could help increase the evidence base and thereby inform the specificity of recommendations and contraindications. In the case of upper-body-led physical activities, less is known about the health risks and benefits, and the extrapolations from the general population are mostly based on lower-body or a combination of upper- and lower-body physical activities. Future research should adopt a functional approach to disability, considering the nature and impact of the impairment on functioning and ability, rather than just the diagnosed condition. This could increase the number of participants available for specific research studies, thereby increasing the strength, and possibly quality, of the evidence. Research-funding mechanisms should prioritize this area to improve knowledge and practice.

There are examples of physical activity studies that have taken a functional approach to disability, incorporating populations with a wide range of health conditions, levels of disability (from none to extremely high), and impairment (mental and sensory, voice and speech, neuromusculoskeletal and movement related). These studies utilized the WHO Disability Assessment Schedule ${ }^{23}$ to assess levels of disability and the International Classification of Functioning Disability and Health, ${ }^{3}$ enabling examinations of physical activity according to functioning. ${ }^{24,25}$ These tools can be used to support a functional approach to the classification of disability in future research studies.

During the development of the guidelines, it was critical to use language that was inclusive and that effectively reflected the evidence for the reviewed outcomes, a point emphasized in the public consultation and peer-review process. One important example concerns sedentary behavior. According to Tremblay et al, ${ }^{26}$ sedentary behavior is sitting, lying, or reclining with low energy expenditure; however, it is common to colloquially equate "sedentary time" with "sitting time." The terms "sitting" or "sit" in messages such as "move more, sit less," or "standing instead of sitting" may be more accessible to the wider public than the term "sedentary," but could lead to misinterpretation of the recommendations and of the underlying evidence base if energy expenditure is not also emphasized. Sitting while cycling, canoeing, or propelling a wheelchair are, of course, not sedentary behaviors. Hence, "sit-less" messages are not considered to be inclusive, especially for people with limited mobility, who sit or lie down all day but can still do light- or high-intensity physical activities while sitting or lying down. The use of universally acceptable language across all recommendations was a priority of the GDG and should remain a priority for stakeholders as they communicate about these new guidelines at a country level.

\section{Mainstreaming Inclusion}

There is increasing acceptance that we need to do more to address disability inclusion in physical activity and sedentary behavior research, policy, and practice, not as a niche and distinct area, but through universal design and mainstreaming. Universally, for people living with disability and those without, the opportunity to sustain participation in physical activity and limit sedentary behavior involves a combination of factors that go far beyond the 


\section{Box 1: The WHO Guidelines on Physical Activity and Sedentary Behavior for Children and Adolescents Living With Disability ${ }^{16}$}

Children and adolescents (aged 5-17 y) living with disability

It is recommended that:

- Children and adolescents living with disability should do at least an average of 60 minutes per day of moderate to vigorous intensity, mostly aerobic, physical activity, across the week.

Strong recommendation, moderate certainty evidence

- Vigorous-intensity aerobic activities, as well as those that strengthen muscle and bone should be incorporated at least 3 days a week.

Strong recommendation, moderate certainty evidence

Good practice statement:

- Doing some physical activity is better than doing none.

- If children and adolescents living with disability are not meeting these recommendations, doing some physical activity will bring benefits to health.

- Children and adolescents living with disability should start by doing small amounts of physical activity and gradually increase the frequency, intensity, and duration over time.

- There are no major risks for children and adolescents living with disability engaging in physical activity when it is appropriate to an individual's current activity level, health status, and physical function; and the health benefits accrued outweigh the risks.

- Children and adolescents living with disability may need to consult a health care professional or other physical activity and disability specialist to help determine the type and amount of activity appropriate for them.

In children and adolescents, higher amounts of sedentary behavior are associated with the following poorer health outcomes: increased adiposity, poorer cardiometabolic health, fitness, behavioral conduct/prosocial behavior, and reduced sleep duration.

It is recommended that:

- Children and adolescents living with disability should limit the amount of time spent being sedentary, particularly the amount of recreational screen time.

Strong recommendation, low-certainty evidence

individual to a broader set of provisions, involving a wide variety of stakeholders and enabling environments. ${ }^{27,28}$

Addressing barriers to physical activity requires comprehensive changes across all 4 strategic objectives of the Global Action Plan on Physical Activity 2018-2030: social norms and attitudes, spaces and places, programs and opportunities, and governance and policy enablers. Providing reasonable accommodations, such as accessible equipment, spaces or programs appropriate to the nature and degree of impairment, and personal and environmental factors, ${ }^{3}$ can enable physical activity participation aligned with ability and help reduce sedentary behavior.

Table 3 outlines 10 key target areas for advancing practice, informed by feedback from the peer-review and public consultation processes, deliberations of the GDG, the policy and human rights context, the International Classification of Functioning Disability and Health, and the Global Action Plan on Physical Activity. The target areas are globally relevant to a broad range of stakeholders who are involved in creating supportive environments for physical activity and limiting sedentary behavior, and they can be tailored to context and situational needs. Stakeholders include policy makers and government officials at national, subnational, and municipal levels; NGOs; research organizations; health service professionals; and exercise and health professionals.

There are financial costs associated with full and effective inclusion, but these inclusive investments benefit all of society, helping to reduce inequity through a proportional universality approach by directing resources to those facing the greatest barriers, as called for in the Global Action Plan on Physical Activity 2018-2030. Investment in inclusive physical activity is empowering and health enhancing and pays dividends by supporting the onward inclusion of people living with disability in other aspects of society and community life..$^{29,30}$

\section{Evidence and Capacity Gaps}

The evidence available to inform these guidelines on physical activity for people living with disability was relatively small, and there is a need to increase the volume of research in this field. The evidence that was available was limited to people with specific conditions or types of impairment, and a limited number of health outcomes, namely, comorbidity, physical function, cognitive function, and quality of life. There was a dearth of research evidence on the critical outcomes that are typically considered in the development of physical activity guidelines, including reduction in all-cause and cause-specific mortality, reduced incidence of cardiovascular disease, reduced incidence of cancer (site-specific), reduced incidence of type 2 diabetes, better bone health, and improvements in adiposity. This is due to people living with disability either not being included in large epidemiological studies, or the sample of people living with disability being too small for subgroup analysis.

A challenge to the inclusion of people living with disability in physical activity practice and in mainstream studies and disabilityspecific studies is the lack of expertise in disability inclusion among many stakeholders working in physical activity. This has resulted in gaps in our knowledge on how best to integrate people living with disability in public health, physical activity, and sedentary behavior interventions. There is a growing body of evidence on the effectiveness of feasible and scalable interventions to promote inclusive physical activity, which should be strengthened for 


\section{Box 2: The WHO Guidelines on Physical Activity and Sedentary Behavior for Adults Living With Disability ${ }^{16}$}

Adults (aged 18 y and over) living with disability

It is recommended that:

- All adults living with disability should undertake regular physical activity.

\section{Strong recommendation, moderate-certainty evidence}

- Adults living with disability should do at least 150-300 min of moderate-intensity aerobic physical activity, or do at least 75-150 min of vigorousintensity aerobic physical activity, or an equivalent combination of moderate- and vigorous-intensity activity throughout the week for substantial health benefits.

\section{Strong recommendation, moderate-certainty evidence}

- Adults living with disability should also do muscle-strengthening activities at moderate or greater intensity that involve all major muscle groups on 2 or more days a week, as these provide additional health benefits.

Strong recommendation, moderate-certainty evidence

- As part of their weekly physical activity, older adults living with disability should do varied multicomponent physical activity that emphasizes functional balance and strength training at moderate or greater intensity on 3 or more days a week, to enhance functional capacity and prevent falls.

\section{Strong recommendation, moderate-certainty evidence}

- Adults living with disability may increase moderate-intensity aerobic physical activity to more than 300 min, or do more than 150 min of vigorous-intensity aerobic physical activity, or an equivalent combination of moderate- and vigorous-intensity activity throughout the week for additional health benefits.

\section{Conditional recommendation, moderate-certainty evidence}

\section{Good practice statement:}

- Doing some physical activity is better than doing none.

- If adults living with disability are not meeting these recommendations, doing some physical activity will bring benefits to health.

- Adults living with disability should start by doing small amounts of physical activity, and gradually increase the frequency, intensity, and duration over time.

- There are no major risks to adults living with disability engaging in physical activity when it is appropriate to the individual's current activity level, health status, and physical function; and when the health benefits accrued outweigh the risks.

- Adults living with disability may need to consult a health care professional or other physical activity and disability specialist to help determine the type and amount of activity appropriate for them.

In adults, higher amounts of sedentary behavior are associated with the following poor health outcomes: all-cause mortality, cardiovascular disease and cancer mortality, and incidence of cardiovascular disease, cancer, and type 2 diabetes.

It is recommended that:

- Adults living with disability should limit the amount of time spent being sedentary and replacing sedentary time with physical activity of any intensity (including light intensity) has health benefits.

\section{Strong recommendation, low-certainty evidence}

- To help reduce the detrimental effects of high levels of sedentary behavior on health, adults living with disability should aim to do more than the recommended levels of moderate-to-vigorous physical activity.

Strong recommendation, low-certainty evidence

people living with disability. There are many freely available resources, programmatic guidance documents, infrastructure considerations, and training opportunities that can be used to mainstream inclusion.

A call for prioritized action on human rights, disability, and intersectional inclusion in recent policy initiatives is a most positive development. The "decade of action" on the Sustainable Development Goals calls for accelerated action to "leave no one behind." It is hoped that, in the aftermath of COVID-19, the attention and investment needed to continue progress will not be eroded. Central to this involves examining how we can continue to leverage human rights instruments, intentions, and reporting to stimulate more inclusive access to physical activity. ${ }^{31}$ This has yet to be fully embraced at national and local levels across communities, schools, workplaces, and in higher education, in order to enable those living with disability to meet the recommendations.

\section{Conclusion}

It is hoped that the publication of the first WHO guidelines on physical activity and sedentary behavior for people living with disability will facilitate concerted efforts across all sectors to bridge gaps in inequalities. At the policy, regulatory, and statutory levels, there is consensus on the need to prioritize and mainstream disability inclusion. However, much work is needed to achieve equity in physical activity opportunities, access, and participation for people living with disability. Multiple stakeholders have a role to play, 
Table 3 Ten Target Areas for Advancing Inclusive Policy, Practice, and Research in Physical Activity and Sedentary Behavior

\begin{tabular}{|c|c|}
\hline 10 target areas & Actions needed \\
\hline 1. Awareness & $\begin{array}{l}\text { Tailored awareness campaigns are needed to draw attention to the inequity experienced by people living with } \\
\text { disability in relation to physical activity. Emphasis on disability as an interaction between a health condition, } \\
\text { personal characteristics, and the environment will help reduce exclusion and point to the broad range of sectors and } \\
\text { actions that are needed to cocreate inclusive physical activity solutions. }\end{array}$ \\
\hline 2. Communication & $\begin{array}{l}\text { Communication campaigns for promoting physical activity and limiting sedentary behavior need to be targeted at } \\
\text { and accessible to people with a wide variety of impairments through a variety of formats and technologies. General } \\
\text { communication messages need to avoid ableist language and sentiment and be universally accessible. }\end{array}$ \\
\hline 3. Environment & $\begin{array}{l}\text { Inclusive access to local amenities, facilities, and services, including green spaces, blue spaces, and networks, may } \\
\text { require new products, technologies, environmental changes, supportive relationships, and inclusive social attitudes. } \\
\text { Safe and connected active transport should be made accessible for people living with disability so that they can } \\
\text { participate more independently where they live, work, play, or go to school. This will help limit sedentary behavior } \\
\text { and increase physical activity among people living with disability. }\end{array}$ \\
\hline 4. Training & $\begin{array}{l}\text { Training and education providers need to supply inclusive practitioners across sectors that impact physical activity } \\
\text { and sedentary behavior to meet the specific needs of people living with disability. Disability awareness training for a } \\
\text { broad range of community stakeholders (professionals to volunteers) would build much-needed understanding and } \\
\text { help reduce the disabling impact of the social and physical environment. }\end{array}$ \\
\hline 5. Partnership & $\begin{array}{l}\text { Facilitating inclusion in and through physical activity is a whole of society issue. Multidisciplinary partnerships } \\
\text { from national policy to local delivery levels are needed to address barriers and facilitators to create opportunities for } \\
\text { participation. They must involve disability service organizations and people living with disability. Dedicated } \\
\text { disability sport inclusion staff, working with disability organizations, can support the inclusion of individuals with } \\
\text { disability in physical activity at community levels. }\end{array}$ \\
\hline 6. Research & $\begin{array}{l}\text { Mechanisms to gather disaggregated data on participation in physical activity, sedentary behavior, and disability are } \\
\text { essential to monitor progress in participation on all levels-local, national, and international. An increased volume } \\
\text { and quality of research exploring barriers and enablers to physical activity and its effects, along the disability } \\
\text { continuum and across the domains of functioning (including life activities and participation), are needed to inform } \\
\text { effective inclusive policy solutions and public health interventions. }\end{array}$ \\
\hline 7. Human rights & $\begin{array}{l}\text { Protecting, respecting, and fulfilling human rights with and for people with disability in and though physical activity } \\
\text { are critical, including targeted interventions for those enduring intersectional discrimination. Increased under- } \\
\text { standing of roles and responsibilities pertaining to human rights is needed and must transfer to inclusive actions, } \\
\text { advocacy, and investments across multiple sectors. }\end{array}$ \\
\hline 8. Programs & $\begin{array}{l}\text { Community-based physical activity programs need to consider disability-specific accommodations (across fully } \\
\text { inclusive to segregated activities) and universal design principles. Facilitating choice in programming is critical, as } \\
\text { is the need to provide opportunities to build positive experiences, beginning early in childhood. }\end{array}$ \\
\hline 9. Investment & $\begin{array}{l}\text { Investment is needed across sectors to advance disability inclusion in and through physical activity, in line with } \\
\text { human rights obligations. It can be tailored according to means through innovative approaches. Appropriate and } \\
\text { effective practical measures, or "reasonable accommodations," such as assistants, carers, and assistive technol- } \\
\text { ogies, should be provided to help people living with disability to be active and to limit sedentary behavior. }\end{array}$ \\
\hline 10. Governance & $\begin{array}{l}\text { Creating inclusive societies requires significant changes at governance and policy levels. Disability inclusion in } \\
\text { public health and physical activity should be mainstreamed through policies and legal frameworks. Partnerships, } \\
\text { finance, and all relevant organs of society should be mobilized to address disability inclusion. With broad } \\
\text { interagency governance structures, physical activity can be a driver of inclusive action in broader society. }\end{array}$ \\
\hline
\end{tabular}

including the research and academic community, in cocreating the inclusive environments that will enable all of us, including those living with disability, to participate in physical activity.

\section{Acknowledgments}

The WHO guideline development process was led by Fiona Bull and Juana Willumsen from the World Health Organization, Geneva, Switzerland. The process was supported by colleagues from several institutions who are acknowledged in the guidelines report. ${ }^{16}$ All members of the WHO Guidelines on physical activity and sedentary behavior GDG contributed to the wording of the final recommendations. The Public Health Agency of Canada and the Government of Norway provided financial support to update the WHO guidelines on physical activity and sedentary behavior.

\section{References}

1. World Health Organization. World Disability Report. Geneva, Switzerland: World Health Organization; 2011.

2. United Nations. Convention on the Rights of Persons with Disabilities (CRPD). New York, NY: United Nations; 2006.

3. World Health Organisation. Towards a Common Language for Functioning, Disability and Health: ICF. Geneva, Switzerland: World Health Organization; 2002.

4. World Health Organization. WHO Global Disability Action Plan 2014-2021. Better Health for all People With Disability. Geneva, Switzerland: World Health Organization; 2015.

5. Cieza A, Sabariego C, Bickenbach J, et al. Rethinking disability. BMC Med. 2018;16(1):14. PubMed ID: 29370847 doi:10.1186/s12916-017$1002-6$ 
6. United Nations Development Programme. Sustainable Urbanization Strategy: UNDP's Support to Sustainable, Inclusive and Resilient Cities in the Developing World. New York, NY: United Nations Development Programme; 2016.

7. United Nations General Assembly. Sport for Development and Peace Sport: A Global Accelerator of Peace and Sustainable Development for All. Report of the Secretary-General. A/75/155. New York, NY: United Nations General Assembly; 2020.

8. Institute for Health Metrics and Evaluation (IHME). Findings from the Global Burden of Disease Study 2017. Seattle, WA: IHME; 2018.

9. Public Health England. Physical Activity for General Health Benefits in Disabled Adults. London, England: Public Health England; 2018.

10. Lee I-M, Shiroma EJ, Lobelo F, Puska P, Blair SN, Katzmarzyk PT. Effect of physical inactivity on major non-communicable diseases worldwide: an analysis of burden of disease and life expectancy. Lancet. 2012;380(9838):219-229. doi:10.1016/S0140-6736(12)61031-9.

11. Rimmer JH, Marques AC. Physical activity for people with disabilities. Lancet. 2012;380(9838):193-195. doi:10.1016/S0140-6736(12)61028-9

12. World Health Organization. Global Action Plan on Physical Activity 2018-2030: More Active People for a Healthier World. Geneva, Switzerland: World Health Organization; 2018.

13. United Nations. Transforming Our World: The 2030 Agenda for Sustainable Development. New York, NY: United Nations; 2015.

14. UNESCO. Report of the Sixth international conference of ministers and senior officials responsible for physical education and sport (MINEPS VI). Annex 1 Kazan Action Plan. SHS/2017/PI/H/14 REV. Paris. September 2017. Adopted on July 14-15, 2017. https://unesdoc. unesco.org/ark:/48223/pf0000252725. Accessed June 20, 2020.

15. UN General Assembly. A/73/325. Strengthening the global framework for leveraging sport for development and peace. Report of the Secretary-General. August 14, 2018. https://undocs.org/en/A/73/ 325. Accessed June 20, 2020.

16. World Health Organization. Guidelines on Physical Activity and Sedentary Behaviour. Geneva, Switzerland: World Health Organization; 2020.

17. World Health Organization. WHO Handbook for Guideline Development. 2nd ed. Geneva, Switzerland: World Health Organization; 2014.

18. World Health Organization. Global Recommendations on Physical Activity for Health. Geneva, Switzerland: World Health Organization; 2010.

19. Bull F, Al-Ansari SS, Biddle S, et al. World Health Organization 2020 guidelines on physical activity and sedentary behaviour. Br J Sports Med. 2020;54(24):1453. doi:10.1136/bjsports-2020-102955

20. U.S. Department of Health and Human Services. Physical Activity Guidelines Advisory Committee Scientific Report. Washington, DC: U.S. Department of Health and Human Services; 2018.
21. Guyatt GH, Oxman AD, Vist GE, et al. GRADE: an emerging consensus on rating quality of evidence and strength of recommendations. BMJ. 2008;336(7650):924-926. PubMed ID: 18436948 doi:10.1136/bmj. 39489.470347.AD

22. Dempsey PC, Friedenreich C, Leitzmann M, et al. Global public health guidelines on physical activity and sedentary behaviour for people living with chronic conditions: a call to action. J Phys Act Health. 2020. doi:10.1123/jpah.2020-0525

23. Ustun TB, Kostanjesek N, Chatterji S, Rehm J. Measuring Health and Disability : Manual for WHO Disability Assessment Schedule (WHODAS 2.0). Geneva, Switzerland: World Health Organization; 2010.

24. Carson V, Hunter S, Kuzik N, et al. Systematic review of sedentary behaviour and health indicators in school-aged children and youth: an update. Appl Physiol Nutr Metab. 2016;41(6)(suppl 3):S240-S265. doi:10.1139/apnm-2015-0630

25. Ćwirlej-Sozańska A, Sozański B, Wiśniowska-Szurlej A, Wilmowska-Pietruszyńska A, Kujawa J. Assessment of disability and factors determining disability among inhabitants of South-Eastern Poland aged 71-80 years. Biomed Res Int. 2018;2018:3872753. PubMed ID: 30018979 doi:10.1155/2018/3872753

26. Tremblay MS, Aubert S, Barnes JD, et al. Sedentary Behavior Research Network (SBRN)-terminology consensus project process and outcome. Int J Behav Nutr Phys Act. 2017;14(1):75. PubMed ID: 28599680 doi:10.1186/s12966-017-0525-8

27. Bauman AE, Reis RS, Sallis JF, Wells JC, Loos RJ, Martin BW. Correlates of physical activity: why are some people physically active and others not? Lancet. 2012;380(9838):258-271. doi:10. 1016/S0140-6736(12)60735-1

28. Martínez-Andrés M, Bartolomé-Gutiérrez R, Rodríguez-Martín B, Pardo-Guijarro MJ, Garrido-Miguel M, Martínez-Vizcaíno V. Barriers and facilitators to leisure physical activity in children: a qualitative approach using the socio-ecological model. Int $J$ Environ Res Public Health. 2020;17(9):3033. doi:10.3390/ijerph17093033

29. UNDESA. Disability and sport. https://www.un.org/development/ desa/disabilities/issues/disability-and-sports.html. n.d. Accessed September 1, 2020.

30. United Nations General Assembly. Seventy-fifth session. Sport for development and peace Sport: a global accelerator of peace and sustainable development for all Report of the Secretary-General. A/75/155. July 2020. https://digitallibrary.un.org/record/3879376? $\ln =$ en. Accessed August 29, 2020.

31. UNESCO. General Conference, 40th, 2019 [1197]. Report of the Intergovernmental Committee for Physical Education and Sport (CIGEPS) 2018-2019. https://unesdoc.unesco.org/ark:/48223/pf0000369231. Accessed June 5, 2020. 